Article original

\title{
Bilan des flux de matières particulaires et dissoutes du Sassandra à Gaoulou pont (Côte d'Ivoire)
}

\author{
Lako AgBrI $^{1}$, Siaka B. BAmBA ${ }^{2}$, Inza DoumouYA ${ }^{1 *}$ \& Issiaka SAVANE ${ }^{1}$, \\ 'Laboratoire Géosciences et Environnement (LGE), UFR Sciences et Gestion de l'Environnement (SGE), Université d'Abobo-Adjamé, \\ UFR SGE 02 BP 801 Abidjan 02 \\ ${ }^{2}$ Centre de Recherches Océanologiques, Rue des pêcheurs, BP V 18 Abidjan - Cote d'Ivoire. \\ *Auteur pour les correspondances (E-mail : doumouya_inza@yahoo.fr) \\ Reçu le 21-02-2008, accepté le 21-06-2010
}

\section{Résumé}

Ce travail a pour objectif l'estimation des apports du fleuve Sassandra. C'est dans ce cadre que les flux de matières particulaires et dissoutes ont été mesurés au cours de l'année 2003 sur le fleuve Sassandra, à la station hydrométrique de Gaoulou. La méthodologie adoptée a consisté à déterminer d'abord les concentrations de matières transportées chaque semaine. Les flux ont été ensuite calculés à l'aide de la relation entre les concentrations et les débits du fleuve. Le flux spécifique total annuel est de $12,9 \mathrm{t} / \mathrm{km}^{2} / \mathrm{an}$. La matière organique représente $55 \%$ de la matière totale en suspension, alors que la matière minérale dissoute constitue $69 \%$ des matières totales transportées. Ces quantités de matières correspondent à des flux spécifiques de $5,2 \mathrm{t} / \mathrm{km}^{2} / \mathrm{an}$ pour les matières en suspension, $7,7 \mathrm{t} / \mathrm{km}^{2} /$ an pour les matières dissoutes. $70 \%$ du transport solide a lieu en juin, septembre et octobre. Mots clés : Sassandra, bassin versant, débit spécifique, érosion, flux de matières, régime hydrologique.

\begin{abstract}
Balance of solid and dissolved matters transported by Sassandra river at Gaoulou bridge (Côte d'Ivoire)

The purpose of this study is to estimate the flow of matters transported by Sassandra river. To execute this program, the flow of matters of Sassandra river has been collected during 2003 year, at Gaul hydraulic station. Methodology adopted consist to determine before matters concentration for each week. The flow of matter has been calculated by the relationship between concentration and water discharge. Specific annual flow rate is $12,9 \mathrm{t} / \mathrm{km}^{2} / \mathrm{an}$ for the set of the elated matters. The organic matter represents $55 \%$ of the suspended matter whereas the matter dissolved is $69 \%$ of the elated matters. Fluxes of matters correspond to the specific debits of $5,2 \mathrm{t} / \mathrm{km}^{2} / \mathrm{an}$ for the suspended matters, $7,7 \mathrm{t} / \mathrm{km}^{2} / \mathrm{an}$ for the dissolved matters. $70 \%$ of the strong transportation take place in June, September and October.
\end{abstract}

Key words: Sassandra, catchment's area, specific flow, erosion, flow of matters, hydrologic system.

\section{Introduction}

Les fleuves constituent la principale source du transfert de matière particulaire et dissoute des continents vers les océans. Les flux de ces matières nous renseignent sur les phénomènes d'érosion et de sédimentation qui affectent le bassin versant (Holeman, 1968). La connaissance 
des quantités et de la nature des matières transportées par les cours d'eau, est un atout à la fois, pour la gestion du bassin versant, et de l'environnement littorale. Plusieurs travaux concernant les apports fluviaux ont été réalisés à travers le monde par divers auteurs : Livingstone (1963), Wailling (1977), Gac (1980), Probst (1983), Dupraz (1984), Bamba (1987), puis Orange (1995) et Orange et al. (1996), Droux et al. (2003), Gruau et al. (2004), Bustillo (2005), Mamoudou (2008). Tous ces travaux ont montré que l'étude du transport particulaire et dissous permet d'apprécier les processus d'érosion et de sédimentation (dans les retenues par exemple), et les phénomènes de pollution des cours d'eau.

En Côte d'Ivoire, le bassin versant du Bandama a fait l'objet d'études concernant le transport particulaire et dissous. En effet, ce bassin versant a été étudié par Mangin et al. (1966), Lenoir (1972), Monnet (1972), Koffi et al. (1987) et Bamba et al. (2004). Le bassin versant du Sassandra subit de fortes pressions anthropiques du fait des activités agricoles. Les pratiques culturales, notamment l'utilisation de produits phytosanitaires et autres engrais peuvent avoir d'énormes conséquences, notamment l'érosion et la dégradation de la qualité des ressources en eau (Adamiade, 2004). Une meilleure appréciation de ces deux phénomènes sur ce bassin versant justifie le choix porté sur le fleuve Sassandra (Agbri, 2006). Deuxième bassin versant ivoirien par sa superficie $\left(75000 \mathrm{~km}^{2}\right.$ dont $8000 \mathrm{~km}^{2}$ sont situés hors du territoire ivoirien) après celui du Bandama, le bassin versant du Sassandra est situé entre $5^{\circ}$ et $9^{\circ} 50$ de latitude nord, et $6^{\circ} 50$ et $8^{\circ}$ de longitude ouest (Figure 1). II comporte au Sud et au Centre, une zone forestière, puis au Nord, une zone de savane. Le contexte géologique du bassin versant est dominé par les roches libériennes (migmatites et gneiss) et des granitoïdes éburnéens : granites, granodiorites (Tagini, 1971) ; la partie sud est constituée de formations à texture plus schisteuses qui sont des migmatites à biotite (Camil, 1984). La couverture pédologique, est à dominante ferrallitique plus ou moins saturée et indurée (Kadio, 1989).

Cet article a pour objet la quantification et le suivi saisonnier des flux de matières exportées au niveau de ce bassin versant, par le fleuve Sassandra à Gaoulou pont.

\section{Matériel et méthodes}

\subsection{Matériel}

Les prélèvements ont été réalisés à l'aide d'une bouteille à renversement. A chaque prélèvement, les échantillons ont été transférés dans des bouteilles en plastique, puis stockés dans une glacière, à une température très basse (inférieure à $4{ }^{\circ} \mathrm{C}$ ). Les échantillons ont été ensuite ramenés au laboratoire pour analyse. La filtration de l'eau en vue de recueillir les matières en suspension a été réalisée sur du papier Wattman GF/F ; une étuve a servi au séchage du produit recueilli.

\subsection{Méthodologie}

\subsection{1-Site de prélèvement}

De janvier à décembre 2003, les prélèvements d'eau ont eu lieu à la station de Gaoulou Pont sur le Sassandra (Figure 1). Cette station hydrologique, située à environ 15 kilomètres de l'océan Atlantique, est considérée comme l'exutoire du bassin versant car elle intègre $94 \%$ de sa superficie, ce qui justifie le choix porté sur ce site pour la quantification des matières déversées par le Sassandra au niveau de l'océan Atlantique.

\subsubsection{Mode opératoire pour les prélèvements}

II existe de nombreuses techniques de mesures des flux détritiques d'origine continentale. Les plus sophistiquées associent simultanément sur toute la section du fleuve les mesures de turbidité et de la vitesse du courant (Billon, 1968). La technique de collecte d'échantillons à hauteur d'une section de mesure est celle que nous avons utilisée. Elle consiste en des prises d'eau d'un litre à l'aide d'une bouteille à renversement en un point de la section mouillée. Dans le cadre de cette étude, les échantillons ont été prélevés à l'échelle hebdomadaire, ce qui représente 52 échantillons par an. La concentration mensuelle de la matière considérée est la moyenne des 4 mesures hebdomadaires.

\subsubsection{Détermination des matières en suspension}

C'est la méthode de filtration qui a été utilisée pour la détermination des matières en suspension présentes dans les eaux. La détermination de la 
charge solide est obtenue après filtration d'un volume $V$ d'échantillon sur papier Wattman GF/F de porosité $0,45 \mu \mathrm{m}$, puis séchage à l'étuve, à 105 ${ }^{\circ} \mathrm{C}$ pendant une heure.

Soit :

$M_{1}$ la masse en mg du filtre avant filtration ;

$M_{2}$ la masse du filtre après filtration et séchage ; $\mathrm{V}$ le volume d'eau filtré en litre.

La quantité de matières en suspension est donnée par l'expression suivante :

$$
\text { M.E.S. }(m g / L)=\left(M_{2}-M_{1}\right) / V
$$

Les produits séchés à l'étuve, et ayant servi pour la quantification des matières en suspension ont été ensuite calcinés entre 450 et $500^{\circ} \mathrm{C}$ pendant deux heures pour brûler la totalité de la matière organique contenue dans les matières en suspension. Ces échantillons sont à nouveau pesés, ce qui donne la masse de matière inorganique (matière sèche) restée sur le filtre. La différence de masse entre les matières en suspension et les matières sèches correspond à la masse de matières organiques particulaires contenues dans les matières en suspension.

\subsubsection{Dosage des cations et anions majeurs}

Les cations $\left(\mathrm{K}^{+}, \mathrm{Ca}^{2+}, \mathrm{Na}^{+}, \mathrm{Mg}^{2+}\right)$ ont été dosés avec un spectrophotomètre d'absorption atomique dans une flamme air-acétylène oxydante. Cette méthode utilise la propriété qu'ont les atomes neutres à absorber à une certaine longueur d'onde un quantum d'énergie.

La silice a été dosée selon la méthode colorimétrique. Les mesures ont été faites au spectrophotomètre à une longueur d'onde de 610 $\mathrm{nm}$. L'anhydride silicique donne en effet avec le molybdate d'ammonium à $\mathrm{pH} \mathrm{1,2} \mathrm{une} \mathrm{coloration}$ jaune due à l'acide silico-molybdique, ce dernier est réduit en anhydride silico-molybdique de coloration bleue.

Les ions chlorures ont été dosés selon la méthode de Charpenuer-Vollard. Ces ions contenus dans une solution de volume connu, ont été précipités en présence d'acide nitrique par un excès de nitrate d'argent titré. L'excès de sel argentique a été déterminé par une solution titrée de sulfocyanure d'ammonium en présence d'alun de fer.

Les sulfates ont été dosés par la méthode Néphélométrique qui consiste à précipiter en milieu chlorhydrique les sulfates à l'état de sulfate de baryum. Le précipité ainsi obtenu a été stabilisé à l'aide d'une solution de " tween 20" ou de polyvinyl-pyrrolidine. Les suspensions homogènes ont été mesurées au spectromètre à $650 \mathrm{~nm}$.

\subsubsection{Méthodes de calcul des flux de matières}

Les méthodes de calculs des flux de matières en suspension sont nombreuses (méthodes stochastiques ou interpolées, méthodes déterministes ou extrapolées) (Mamoudou, 2008). La méthode stochastique est recommandée car adaptée à toutes les situations hydrologiques et surtout aux éléments dont les concentrations varient avec les débits (Garcia, 2007). Cette méthode utilise les relations débits-concentrations (Wailling, 1977; Balland, 1983; Dupraz, 1984; Meybeck, 1984; Bamba et al., 2004). Le flux moyen annuel de sédiments (Ta) transportés est calculé à partir des débits moyens mensuels grâce à la relation :

$$
\mathrm{Ta}=86,4 \cdot 10^{-3} \sum 1^{12} \text { (Cm.Qm) }
$$

Ta : flux annuel en tonnes

$\mathrm{Cm}$ : concentration moyenne mensuelle $(\mathrm{mg} / \mathrm{L})$

$\mathrm{Qm}$ : débit moyen mensuel $\left(\mathrm{m}^{3} / \mathrm{s}\right)$

Le tonnage spécifique ou flux spécifique exprimé en tonne par kilomètre carré $\left(\mathrm{t} / \mathrm{km}^{2}\right)$, est obtenu en divisant le flux total annuel par la surface totale du bassin versant. Ceci permet de comparer les valeurs obtenues sur différents bassins de tailles différentes. 


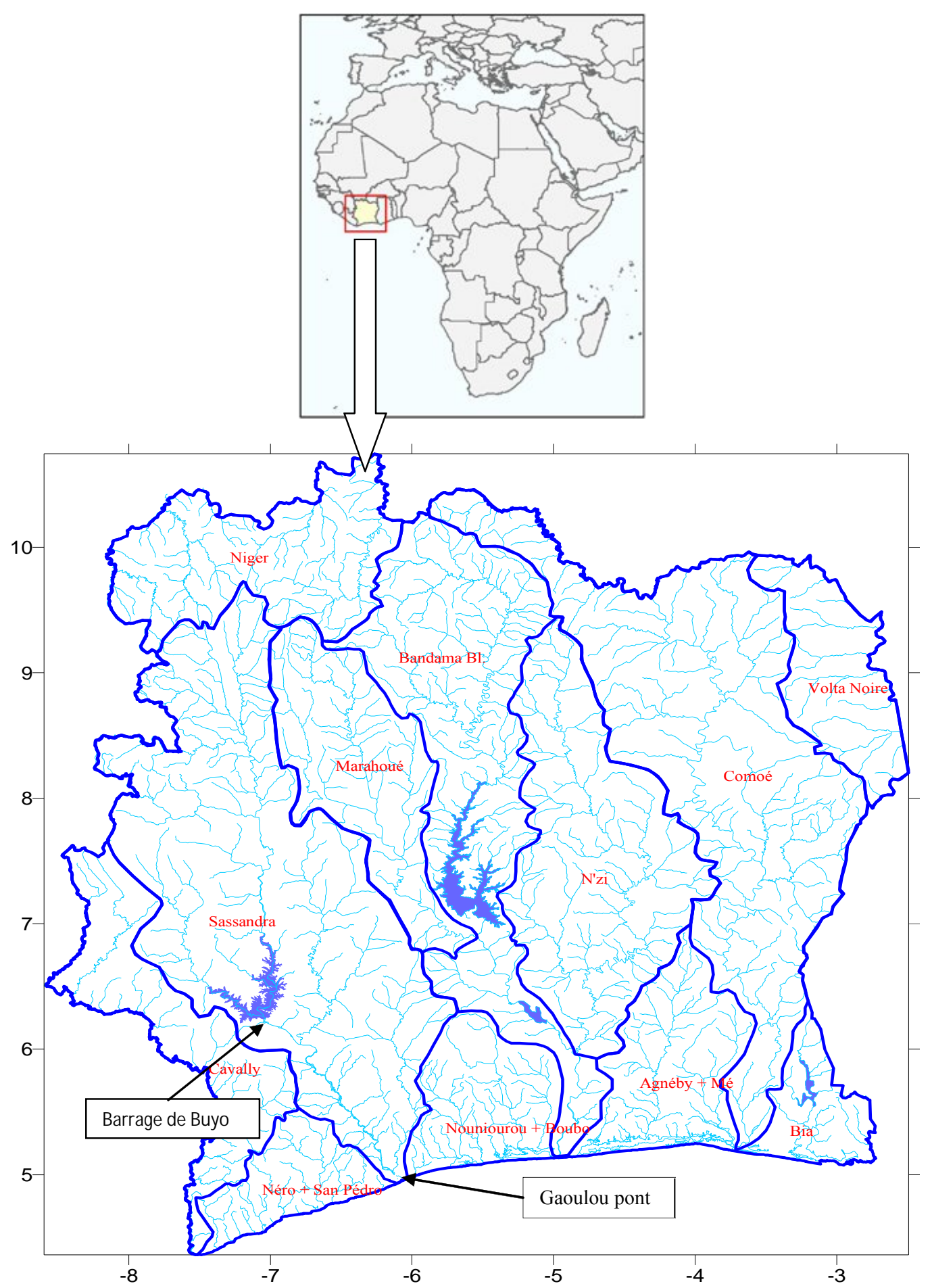

Figure 1 : Bassin versant du Sassandra (in annuaire hydrologique 1983) 


\section{Résultats}

\subsection{Débits du fleuve Sassandra}

Les débits observés à Gaoulou se traduisent par deux périodes de crue : une première crue en juin et la seconde en septembre-octobre. Sur la période 1981-2000, on constate que la première crue est assez faible $\left(513,4 \mathrm{~m}^{3} / \mathrm{s}\right)$ par rapport à la seconde $\left(1163,53 \mathrm{~m}^{3} / \mathrm{s}\right)$. Les débits de l'année 2003 semblent peu différents de ceux de la période sus-citée car le débit du mois de juin est de $1029,73 \mathrm{~m}^{3} / \mathrm{s}$, tandis que celui du mois de septembre est de $1265,57 \mathrm{~m}^{3} / \mathrm{s}$ (Tableau 1).

Tableau 1 : Bilan annuel des flux de matières particulaires du fleuve Sassandra

\begin{tabular}{|c|c|c|c|c|c|c|}
\hline Mois & $\begin{array}{l}\text { MES } \\
(\mathrm{mg} / \mathrm{l})\end{array}$ & $\begin{array}{c}\mathrm{MO} \\
(\mathrm{mg} / \mathrm{l})\end{array}$ & $\begin{array}{c}\text { Débits } 2003 \\
\left(\mathrm{~m}^{3} / \mathrm{s}\right)\end{array}$ & $\begin{array}{c}\text { Débits } 1981-2000 \\
\left(\mathrm{~m}^{3} / \mathrm{s}\right)\end{array}$ & $\begin{array}{c}\text { Flux } \\
\text { MES (t) }\end{array}$ & $\begin{array}{l}\text { Flux } \\
\mathrm{MO}(\mathrm{t})\end{array}$ \\
\hline janvier & 7,12 & 2,68 & 301,43 & 302,4 & 5748,3 & 2163,7 \\
\hline février & 8,9 & 4,7 & 414,3 & 217,82 & 8920,2 & 4710,7 \\
\hline mars & 9,3 & 3,9 & 367,48 & 216,33 & 9153,6 & 3838,6 \\
\hline avril & 13,2 & 7 & 403,57 & 221,74 & 13807,9 & 7322,4 \\
\hline mai & 15,12 & 2,96 & 442,81 & 264,26 & 17932,6 & 3510,6 \\
\hline juin & 17,4 & 7,8 & 1029,73 & 485,91 & 46441,6 & $\begin{array}{c}20818 \\
7\end{array}$ \\
\hline juillet & 12,16 & 5,36 & 474,19 & 467,01 & 15444 & 6807,6 \\
\hline août & 8,93 & 2,67 & 289,55 & 684,88 & 6925,5 & 2070,7 \\
\hline septembre & 19,4 & 11,7 & 1265,57 & 1163,53 & 63638,9 & $\begin{array}{c}38380 \\
2\end{array}$ \\
\hline octobre & 45,55 & 31,15 & 1264,16 & 1092,09 & 154228,9 & $\begin{array}{c}105471 \\
, 6\end{array}$ \\
\hline novembre & 20,1 & 7,15 & 390,77 & 650,88 & 20358,8 & 7242 \\
\hline décembre & 9,9 & 2,3 & 287,97 & 393,20 & 7636 & 1774 \\
\hline Total annuel (t) & & & & & 370263 & $\begin{array}{c}204.11 \\
1 \\
\end{array}$ \\
\hline $\begin{array}{l}\text { Flux spécifique } \\
\text { (t//kmªn) }\end{array}$ & & & & & 5,2 & 2,9 \\
\hline
\end{tabular}

\subsection{Les matières en suspension et les matières dissoutes}

Les concentrations mensuelles des matières en suspension varient de $7,12 \mathrm{mg} / \mathrm{L}$ en janvier à $45,55 \mathrm{mg} / \mathrm{L}$ en juin, et de $2,3 \mathrm{mg} / \mathrm{L}$ en décembre à $31,15 \mathrm{mg} / \mathrm{L}$ en octobre pour les matières organiques (Tableau 1). Ces variations montrent que les périodes de crue correspondent aux fortes concentrations, alors que les faibles concentrations sont les périodes de décrue (Figure 2b).
Les éléments minéraux dosés dans les échantillons prélevés sont consignés dans le tableau 2. L'observation des concentrations montre que la silice est largement majoritaire car elle représente $54 \%$ des matières minérales dissoutes. Viennent ensuite par ordre d'importance, le sodium, le chlore, le potassium et le magnésium. En terme d'évolution, certaines concentrations augmentent avec la crue $\left(\mathrm{Mg}^{++}\right.$, $\mathrm{Na}^{+}$) (Figure 2a), tandis que d'autres semblent evoluer de manière aléatoire $\left(\mathrm{SiO}_{2}, \mathrm{~K}^{+}, \mathrm{Cl}^{-}, \mathrm{Ca}^{++}\right)$ (Figure 2c et 2d). 


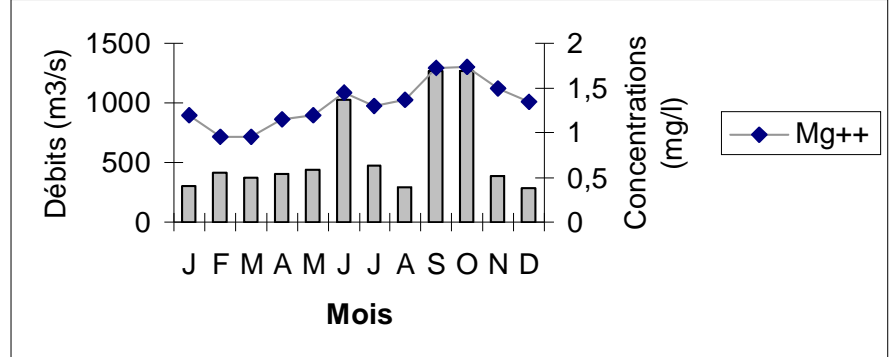

a) magnésium

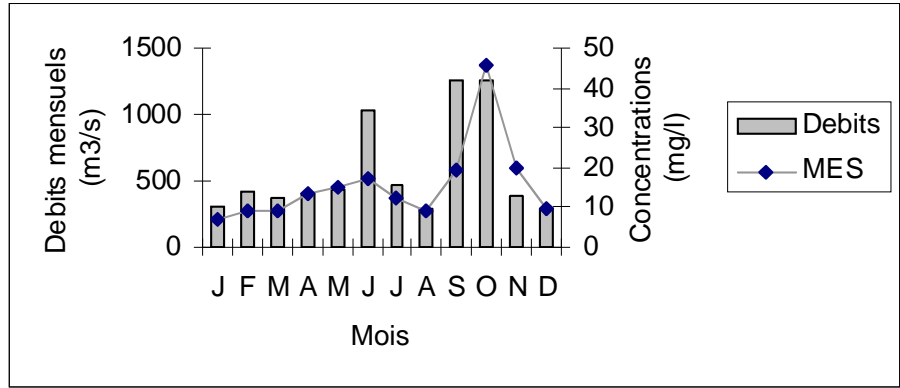

b) Matières en suspension.

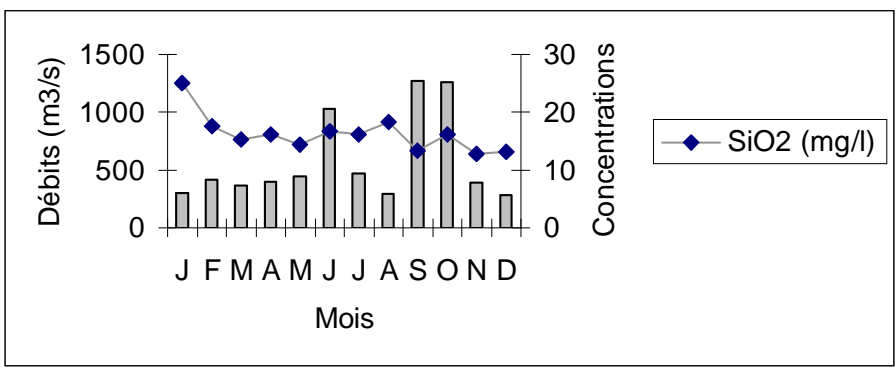

c) silice.

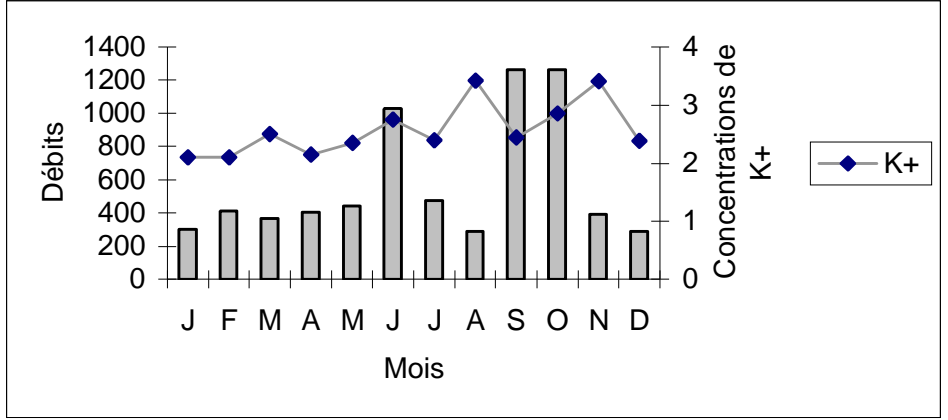

d) Potassium.

Figure 2 : Evolution comparée des matières particulaires et dissoutes par rapport aux débits 
Tableau 2 : Composition chimique moyenne des eaux du fleuve Sassandra en mg/l.

\begin{tabular}{lccccccc}
\hline Mois & $\mathrm{SiO}_{2}$ & $\mathrm{Cl}^{-}$ & $\mathrm{Ca}^{++}$ & $\mathbf{M g}^{++}$ & $\mathrm{Na}^{+}$ & $\mathrm{K}^{+}$ & $\mathbf{F e}^{2+}$ \\
\hline janvier & 25,1 & 1,28 & 1,7 & 1,2 & 4,5 & 2,1 & 0,03 \\
février & 17,65 & 1,44 & 1,75 & 0,95 & 4,1 & 2,1 & 0,03 \\
mars & 15,3 & 2,56 & 1,8 & 0,95 & 4,25 & 2,5 & 0,02 \\
avril & 16,1 & 1,6 & 1,55 & 1,15 & 4,6 & 2,15 & 0,03 \\
mai & 14,38 & 2,8 & 2 & 1,2 & 5,13 & 2,35 & 0,42 \\
juin & 16,68 & 4,8 & 1,95 & 1,45 & 6,25 & 2,75 & 0,02 \\
juillet & 16,08 & 3,36 & 2,45 & 1,3 & 6,35 & 2,4 & 0,2 \\
août & 18,2 & 3,97 & 1,08 & 1,37 & 4,8 & 3,42 & 0,05 \\
septembre & 13,4 & 2,84 & 1 & 1,72 & 3,6 & 2,44 & 0,08 \\
octobre & 16,21 & 1,42 & 1,72 & 1,73 & 4,1 & 2,85 & 0,2 \\
novembre & 12,8 & 2,9 & 1,01 & 1,49 & 4,8 & 3,41 & 0,15 \\
décembre & 13,2 & 3,19 & 1,38 & 1,35 & 3,7 & 2,38 & 0,7 \\
TOTAL & $\mathbf{1 9 5 , 1}$ & $\mathbf{3 2 , 1 6}$ & $\mathbf{1 9 , 3 9}$ & $\mathbf{1 5 , 8 6}$ & $\mathbf{5 6 , 1 8}$ & $\mathbf{3 0 , 8 5}$ & $\mathbf{1 , 9 3}$ \\
Moyenne & $\mathbf{1 6 , 2 6}$ & $\mathbf{2 , 6 8}$ & $\mathbf{1 , 6 2}$ & $\mathbf{1 , 3 2}$ & $\mathbf{4 , 6 8}$ & $\mathbf{2 , 5 7}$ & $\mathbf{0 , 1 6}$ \\
Pourcentage (\%) & $\mathbf{5 4 , 0}$ & $\mathbf{9 , 0}$ & $\mathbf{5 , 3}$ & $\mathbf{4 , 3}$ & $\mathbf{1 5 , 5}$ & $\mathbf{8 , 5}$ & $\mathbf{0 , 5}$ \\
\hline
\end{tabular}

\subsection{Bilan des flux de matières}

Au cours de la période d'étude, les flux de matières dissoutes restent toujours supérieurs à ceux des matières en suspension, à l'exception de la seconde période de crue du fleuve (septembre-octobre) au cours de laquelle le transport en suspension atteint sa valeur extrême (Tableau 1).

Avec un module annuel moyen de $577,63 \mathrm{~m}^{3} / \mathrm{s}$, la quantité de matière exportée est estimée à
$0,37.10^{6} \mathrm{t}$ pour les matières en suspension (Tableau 1) et $0,29.10^{6} \mathrm{t}$ pour la silice (Tableau $3)$. Les débits spécifiques respectifs sont de 5,2 $\mathrm{t} / \mathrm{km}^{2} /$ an et $4,1 \mathrm{t} / \mathrm{km}^{2} / \mathrm{an}$. Le bilan annuel qui est de $0,9 \cdot 10^{6} \mathrm{t}$ à Goulou, correspond à un débit spécifique de $12,9 \mathrm{t} / \mathrm{km}^{2} / \mathrm{an}$. Les matières en suspension représentent $40 \%$ du flux total de matières transportées, alors que les matières organiques particulaires représentent $55 \%$ des matières en suspension. Quand à la silice, elle représente $53 \%$ du flux de matières minérales dissoutes (Tableau 3). 
Tableau 3 : Bilan annuel (tonnes) des flux de matières dissoutes du fleuve Sassandra

\begin{tabular}{lccccccc}
\hline Mois & $\mathbf{S i O}_{2}$ & $\mathbf{C l}^{-}$ & $\mathbf{C a}^{++}$ & $\mathbf{M g}^{++}$ & $\mathbf{N a}^{+}$ & $\mathbf{K}^{+}$ & TOTAL \\
\hline janvier & 20264 & 1033 & 1372 & 969 & 3633 & 1695 & 28966 \\
février & 17690 & 1443 & 1754 & 952 & 4109 & 2105 & 28323 \\
mars & 15059 & 2520 & 1772 & 935 & 4183 & 2460 & 26929 \\
avril & 16841 & 1674 & 1621 & 1203 & 4812 & 2249 & 28400 \\
mai & 17049 & 3321 & 2372 & 1423 & 6084 & 2787 & 33036 \\
juin & 44520 & 12811 & 5205 & 3870 & 16681 & 7340 & 90427 \\
juillet & 20422 & 4267 & 3112 & 1651 & 8065 & 3048 & 40565 \\
août & 14114 & 3079 & 838 & 1062 & 3722 & 2652 & 27018 \\
septembre & 43957 & 9316 & 3280 & 5642 & 11809 & 8004 & 90750 \\
octobre & 54886 & 4808 & 5824 & 5857 & 13882 & 9650 & 101679 \\
novembre & 12965 & 1013 & 1023 & 1509 & 4862 & 3454 & 26852 \\
décembre & 10181 & 2460 & 1064 & 1041 & 2854 & 1836 & 20978 \\
TOTAL (t) & 288218 & 47745 & 29237 & 26114 & 84696 & 47280 & 523290 \\
Pourcentage (\%) & $\mathbf{5 5}$ & $\mathbf{9}$ & $\mathbf{6}$ & $\mathbf{5}$ & $\mathbf{1 6}$ & $\mathbf{9}$ & $\mathbf{1 0 0}$ \\
Flux spécifique & $\mathbf{4 , 1}$ & $\mathbf{0 , 7}$ & $\mathbf{0 , 4}$ & $\mathbf{0 , 4}$ & $\mathbf{1 , 2}$ & $\mathbf{0 , 7}$ & $\mathbf{7 , 7}$ \\
(t/km /an) & & & & & & & \\
\hline
\end{tabular}

\section{Discussion}

\subsection{Régime hydrologique du Sassandra}

L'observation des débits du fleuve Sassandra Gaoulou, montre que son régime hydrologique s'apparente assez étroitement à celui des précipitations. II en est de même sur l'ensemble de son bassin versant qui est assez allongé du nord au sud. En effet, en amont du barrage de Buyo notamment à Guiglo et Sémien, le régime d'écoulement est caractérisé par une seule alternance de hautes eaux et de basses eaux (un maximum en août-septembre et l'étiage en janvier-février) : c'est un régime hydrologique de montagne, alors qu'en aval à Gaoulou, le régime d'écoulement est caractérisé par deux (2) périodes de hautes eaux dont la première se situe en juin et la seconde en septembreoctobre : c'est un régime équatorial de transition pur (Yapo, 2002). II s'agit d'un régime hydrologique mixte (Musy, 2005) car ce fleuve subit diverses influences du fait de l'étendue de son bassin hydrologique comme d'ailleurs les autres grands fleuves de la Côte d'Ivoire (Bandama, Comoé, Cavally) (Agbri, 2006). Depuis la mise en eau du barrage de Buyo (1981), le fonctionnement naturel du Sassandra est modifié et a conduit la partie aval du barrage à fonctionner de façon artificielle, car les débits aval du cours d'eau sont tributaires des débits lâchés au niveau du barrage. Des études ont mis en évidence l'influence du barrage sur l'hydrologie du Sassandra à environ cent kilomètres en aval, à Soubré (Yapo, 2002). Or, il y a une corrélation très forte entre les débits à Soubré et ceux de Gaoulou $\left(R^{2}=0,997\right)$, ce qui indique que l'influence $d u$ barrage de Buyo se manifeste à Soubré.

\subsection{Variations mensuelles des matières en suspension}

Les fortes concentrations en matières en suspension s'observent pendant les crues (octobre) et les plus faibles lors de la décrue (décembre à mars). Cela peut être dû au fait que les premières pluies lessivent les sols dénudés ou qui ont été labourés ; ces pluies emportent une partie du matériel détritique disponible pour le transport particulaire. Avec la persistance de la pluie, tout le matériel détritique de moindre résistance est totalement emporté. II s'agit d'une évolution classique observée dans la plupart des grands cours d'eau du domaine climatique soudanien (Kattan et al., 1987; Olivry et al., 1989; Probst, 1983 ; Orange et al., 1995). 
La deuxième crue provoque une forte augmentation des teneurs en matières en suspension du cours d'eau à cause du très grand volume d'eau mis en jeu, c'est-à- dire lorsque la contribution du ruissellement superficiel à l'écoulement fluvial est maximale (Probst 1983). En effet, le ruissellement superficiel est l'écoulement responsable de l'érosion mécanique. On peut considérer qu'en dehors des périodes de ruissellement superficiel, les sédiments transportés par les cours d'eau sont arrachés au lit et aux berges. L'érosion des berges est évaluée à $30 \%$ du transport total en suspension dans le cas des fleuves Girou (France), Chari (Tchad) et Sénégal (Bamba, 1987), alors qu'elle représente en moyenne $26 \%$ pour les cours d'eau des EtatsUnis (Bamba et al., 2004). La réponse temporelle des concentrations en matières en suspension dépend de la périodicité de la crue (centennale, biennale), de la position de la crue dans la chronologie des évènements de crues annuelles (première, seconde,...), des caractéristiques propres (pentes) et anthropiques (occupation des sols) du bassin versant et de la gestion des crues (lâchée de barrage) (Mamoudou, 2008).

\subsection{Variations mensuelles des matières dissoutes}

La composition chimique des eaux du Sassandra est marquée par une forte concentration de silice. Cette forte quantité de silice observée est normale car dans la plupart des eaux des rivières en Afrique de l'ouest et centrale (Gac, 1980 ; Meybeck, 1984 ; Martins \& Probst, 1991), les hydrogénocarbonates et la silice sont les deux espèces minérales dissoutes largement majeures. Elles représentent près de 80 $\%$ de la masse minérale totale dissoute (Orange et al., 1995). La silice provient essentiellement de l'altération des minéraux silicatés. L'accumulation des produits d'altération durant la période d'étiage provoque une augmentation des concentrations de silice. Par la suite, durant la période des hautes eaux, les teneurs en silice diminuent (Probst, 1983) car la montée des eaux entraîne une dilution des concentrations en éléments minéraux dissous. Cette diminution des concentrations est également due à la participation de la silice aux cycles nutritifs d'espèces végétales supérieures et planctoniques (Wollast \& Mackenzie, 1983).
Au niveau des éléments minéraux dissous, d'une manière générale, les concentrations mensuelles du sodium sont supérieures à celles des autres ions (potassium, magnésium, chlore et calcium). II existe une assez bonne corrélation entre le magnésium et les débits $\left(R^{2}=0,74\right)$ (figure $\left.2 a\right)$ et à un degré moindre entre le sodium et les débits $\left(R^{2}=0,65\right)$. L'évolution du potassium (figure $2 d$ ) et des autres éléments minéraux se fait sans tendance apparente.

Le magnésium (Figure 2a) et le sodium (à un degré moindre) ont une origine superficielle car ces deux éléments sont liés à la crue. En revanche, l'augmentation des teneurs des autres éléments chimiques à la fois en période d'étiage et pendant les crues, montre qu'ils ont une double origine à la fois profonde et superficielle ; car en période d'étiage, les rivières sont essentiellement alimentées par les eaux issues des nappes souterraines (Probst, 1992).

\subsection{Bilan annuel des matières exportées par le Sassandra}

Au cours de la période d'étude, les flux de matières dissoutes étaient toujours supérieurs à ceux des matières en suspension, à l'exception de la seconde période de crue du fleuve, au cours de laquelle le transport en suspension atteint sa valeur extrême. Le bilan annuel qui est de $0,9.10^{6}$ $\mathrm{t}$, correspondant à un débit spécifique de 12,9 t/ $\mathrm{km}^{2} / \mathrm{an}$, traduit une érosion chimique et mécanique intense comme sur la plupart des bassins humides forestiers. A titre comparatif, le flux moyen annuel de matières apportées à l'Oubangui par les fleuves Uélé, Bili et M'bonou est estimé à $4,8.10^{6}$ t par an, ce qui correspond à un flux spécifique de $13,3 \mathrm{t} / \mathrm{km}^{2} /$ an pour le fleuve Oubangui, avec un flux spécifique dissous de $7,8 \mathrm{t} / \mathrm{km}^{2} /$ an (Orange et al., 1995), tandis que les apports de matières particulaires et dissoutes de la cuvette amazonienne à l'atlantique sont évalués à $0,27.10^{12} t$ de matières dissoutes avec $1,2.10^{9}$ millions de tonnes de matières en suspension (Anonyme, 2005). La plus grande partie du transport de matières a lieu pendant la crue. En effet, $70 \%$ du transport solide a lieu en juin, septembre et octobre pour le Sassandra $\left(26,4.10^{4}\right.$ t/an), alors que dans le cas du Bandama, $68 \%$ du transport soluble s'effectue entre les mois de septembre et novembre à Bafécao (Bamba et al., 2004). Les différents pourcentages de matières 
en suspension et de matières dissoutes varient en général d'un fleuve à l'autre en fonction de la proportion de forêt et de savane sur le bassin versant. La matière organique dissoute croit avec le pourcentage de forêt du bassin versant. En effet, le débit spécifique de matières organiques dissoutes est de 1,3 t/km²/an pour le M'bonou, 3,8 $\mathrm{t} / \mathrm{km}^{2} / \mathrm{an}$ pour l'Uélé et de $5,9 \mathrm{t} / \mathrm{km}^{2} / \mathrm{an}$ pour la Bili (Orange et al., 1995). Le taux de couverture de forêt semi-humide de ces bassins versants est respectivement de $17 \%, 27 \%$ et $44 \%$.

La quantification du transport solide des cours d'eau est le meilleur moyen d'estimer l'intensité de l'érosion mécanique sur les bassins versants. Quant au transport dissous, il permet d'estimer la vitesse moyenne de l'érosion chimique des continents. D'une manière générale, le transport dissous est supérieur au transport en suspension. II représente $59 \%$ dans le cas du Sassandra, alors qu'il est de $66 \%$ dans le cas de l'Oubangui (Orange et al., 1996). Dans le cas spécifique du Sassandra, une partie des matières en suspension serait piégée au niveau de la retenue du barrage de Buyo, ce qui pourrait avoir une influence sur les quantités de matières à l'exutoire du bassin versant.

\section{Conclusion}

Les variations des concentrations mensuelles en matières particulaires et dissoutes du fleuve Sassandra sont importantes, et suivent une évolution bien connue. En effet, l'acquisition de la charge solide par les eaux est progressive au cours de la montée pour atteindre son maximum pendant la crue. Cela traduit la nette relation entre la charge solide et le régime hydrologique du cours d'eau. La charge solide est tributaire du régime des précipitations. Les résultats du transport particulaire et dissous sur le bassin versant du Sassandra, du fait de sa très forte proportion de forêt, sont semblables à ceux d'un bassin forestier en zone tropical humide. Le tonnage annuel de matières exportées par le fleuve (matières en suspension et matières dissoutes) sur la période d'étude correspond à un débit spécifique de 12,9 $\mathrm{t} / \mathrm{km}^{2} / \mathrm{an}$. Ce débit spécifique équivaut à un flux annuel d'environ $0,9.10^{6}$ tonnes, et environ $70 \%$ de ce tonnage annuel est transportée pendant les périodes de crue. Le flux dissous $\left(7,7 \mathrm{t} / \mathrm{km}^{2} / \mathrm{an}\right)$, correspondant à l'érosion chimique du bassin versant, est supérieur au flux particulaire $\left(5,2 \mathrm{t} / \mathrm{km}^{2} /\right.$ an) qui correspond à l'érosion mécanique. Les matières organiques particulaires représentent 55 $\%$ des matières en suspension. L'altération silicatée est très intense, se traduisant par une forte exportation de la silice $\left(4,1 \mathrm{t} / \mathrm{km}^{2} / \mathrm{an}\right)$, qui par ailleurs, représente $53 \%$ de l'ensemble des matières minérales dissoutes.

\section{Remerciements}

Nos remerciements vont à la Direction de la Météorologie Nationale de la SODEXAM et à la Direction de l'Hydraulique humaine qui ont bien voulu mettre à notre disposition respectivement les données pluviométriques couvrant la zone d'étude et les débits du fleuve Sassandra relevés à la station hydrologique de Gaoulou pont.

\section{Références citées}

Agbri L., 2006. Variations et bilans des flux de matières particulaires et dissoutes $d u$ Sassandra à Gaoulou pont (Côte d'lvoire). Mémoire de DEA en Sciences et Gestion de l'Environnement, Université d'AboboAdjamé, 60 pp.

Adamiade V. C., 2004. Influence d'un fosse sur les écoulements rapides au sein d'un versant. Thèse de spécialité Géosciences et Ressources naturelles, mention Hydrologie, Université Pierre et Marie Curie, 216 pp.

Anonyme, 2005. Les écoulements dans la cuvette amazonienne, 8 pp. www.aubresil.net, visité le 17/12/2005.

Balland P., 1983. Méthodologie de quantification des flux de nutriments en rivières. In : IAHS Publication $n^{\circ} 141$, pp 333-345.

Bamba S.B., 1987. Le bilan de l'eau et de la matière dans le haut basin Guinéen du fleuve Gambie. Thèse de Doctorat $3^{\text {eme }}$ cycle, Géologie mention hydrogéologie. Université Cheikh Anta Diop de Dakar, faculté des sciences, $148 \mathrm{pp}$.

Bamba S. B., Abé J., Affian K., Metongo B.S. \& Adingra A., 2004. Transport particulaire et dissous d'un fleuve intertropical: le Bandama (Côte d'Ivoire). In : Bioterre, Revue International Science de la Vie et de la Terre, volume 4. Édition universitaire de Côte d'Ivoire, pp. 37-58. 
Billon B., 1968. Mise au point des mesures de débits solides en suspension, République du Tchad, cahier ORSTOM, série hydrologie 52, pp 3-13.

Bustillo V., 2005. Biogéochimie et hydroclimatologie appliquée à l'aménagement des bassins fluviaux. Modèles de mélanges. Diagnostic et prévision. Cas du Niger, de l'Amazone et de la Garonne. Thèse de Doctorat de l'Institut Polytechnique de Toulouse, spécialité : Sciences Agronomiques, 243 pp.

Camil J., 1984. Pétrographie chronologique des ensembles granulitiques archéens et formations associées de la région de Man (Côte $d^{\prime}$ 'lvoire). Thèse de Doctorat d'Etat ès Sciences naturelles. Université de Cocody, 306 pp.

Droux J.P., Mietton M. \& Olivry J.C. 2003. Flux de matières en zone de savane soudanienne: l'exemple de trois bassins versants maliens représentatifs. Géomorphologie : relief, processus, environnement, volume 9 numéro 2, pp. $99-110$.

Dupraz C., 1984. Bilans des transferts d'eau et d'éléments minéraux dans trois bassins versants comparatifs à végétations contrastées (Mont-Lozère, France). Thèse de Doctorat d'Ingénieur, Géologie appliquée (hydrologie). Université Orléans, 363 pp.

Gac J. Y., 1980. Géochimie du bassin du lac Tchad. Bilan de l'altération, de l'érosion et de la sédimentation. In : Travaux et documents, ORSTOM. Paris, pp.123.

Garcia E. J., 2007. Géochimie d'un fleuve côtier méditerranéen. La têt en Rossillon. Origine et transfert de matières dissoutes et particulaires de la source jusqu'à la mer. Thèse de Doctorat. Université de Perpignan, 222 pp.

Gruau G., Jarde E., Birgand F. \& Novince E. 2004. Pollution des eaux continentales de Bretagne par les matières organiques. Flux, concentrations moyennes et dynamique des transferts dans les bassins versants. Réflexions sur les indicateurs, caractérisations moléculaires des sources. Rapport d'étude, DRASS Bretagne, $110 \mathrm{pp}$.

Holleman J.J., 1968. The sediment yield of major rivers in the world. In: revue scientifique, Water Resources $n^{\circ}$ 4, pp $737-740$.
Kadio E., 1989. Etudes géologiques et géochimiques des occurrences nickélifères supergènes au nord-ouest de Biankouma (Côte d'Ivoire). Thèse de spécialité. Université de Cocody, $270 \mathrm{pp}$.

Kattan Z., GAC J.Y., \& Probst J.L., 1987. Suspended sediment load and mechanical erosion in the Senegal basin, estimation of the surface runoff concentration and relative contributions of channel and slope erosions. Journal Hydrologique 1992, pp 59-76.

Koffi K. P., Abé J., \& Affian K., 1987. Évaluation actuelle du littoral ivoirien (Résultats préliminaires du suivi 1985-1987). Note à diffusion restreinte, novembre 1987, Centre de Recherches Océanologiques.

Lenoir F., 1972. Exportation d'un bassin intertropical :le Bandama (Côte d'lvoire). Influence des conditions hydrologiques. Thèse de Doctorat d'Etat. Université de Nice, 375 pp.

Livingstone D.A., 1963. Chemical composition of rivers and lakes. In: U.S. Geology, Data of geochemistry. pp 1- 64.

Meybeck M., 1984. Les fleuves et le cycle géochimique des éléments. Thèse de Doctorat ès Science et Géologie. Université Paris VI, $558 \mathrm{pp}$.

Mamoudou M., 2008. Dynamique de transfert organique et inorganique le long du continum fluvial de la Garonne: impact de la retenue de Malause. Thèse de Doctorat de l'Université de Toulouse, specilité Agroforesterie, Écosystèmes et Environnement, 198 pp.

Mangin J.P., Lecolle J., Mathieu P., Monnet C., Pinta M. \& Sircoulou J., 1966. Géochimie des eaux naturelles, le transport en solution par un fleuve de Côte d'Ivoire. In : revue académique scientifique $n^{\circ} 262$, Paris. pp $2204-2206$.

Martins O. \& Probst J.L., 1991. Biogechemestry of major African rivers; carbon and mineral transport. In: Biogeochemistry of major world rivers $n^{\circ} 64$, edition scope, pp. 483-508.

Monnet C., 1972. Contribution à l'étude de la dynamique et de la nature des suspensions d'un fleuve intertropical, le Bandama, Côte d'lvoire. Evolution des éléments chimiques des eaux de son estuaire. Thèse de Doctorat d'Etat. Université de Nice, 416 pp. 
Musy A., 2005. Les régimes hydrologiques. Cours d'hydrologie générale, $25 \mathrm{pp}$.

Olivry J.C., Bricquet J.P. \& Thiebaux J.P., 1989. Bilan annuel et variations saisonnières des flux particulaires du Congo à Brazzaville et de l'Oubangui à Bangui. In : Editions La houille blanche 3/4, pp 311-315.

Orange D., 1995. Transport de matières dans un bassin fluvial tropical humide en zone de forêt : I 'Uélé au Zaire. Rapport ORSTOM, Congo. www.mpl.ird.fr/hydrologie/document/ monogras/oubangui/referec/7/7.htm, (visité le 26/09/2005). 10 pp.

Orange D., Olivry J.C. \& Censier C., 1996. Variations et bilans des flux de matières particulaires et dissoutes de l'Oubangui à Bangui (de 1987 à 1992), Rapport ORSTOM www.mpl.ird.fr/ hydrologie/document/monogras/oubangui/ referec/7/7.htm, (visité le 26/09/2005), 6 pp.

Probst J .L., 1983. Hydrologie du bassin de la Garonne. Modèle de mélanges, bilans de l'érosion, exportation des phosphates et des nitrates. Thèse de Doctorat $3 e$ cycle. Université
Paul Sabatier Toulouse, 148 pp.

Probst J.L., 1992. Géochimie et hydrologie de l'érosion continentale. Mécanismes, bilan global actuel et fluctuations au cours des 500 derniers millions d'années. Mémoires Sciences Géologiques 94. Strasbourg, 167 pp.

Tagini B., 1971. Esquisse structurale de la Côte d'Ivoire, essai de géotectonique régional. SODEMI, $302 \mathrm{pp}$.

Walling D.E., 1977. Limitations of the rating curve technique for estimating suspended sediment loads with particular reference to British rivers. In IAHS Publication n¹22, pp. 34 -48.

Wollast R. \& Mackenzie F.T., 1983. The global cycle of silica. In: Silicon geochemistry and biogeochemistry, Edition Aston Academic Press, London, pp.39-76.

Yapo O. B., 2002. Contribution à l'évolution de l'état de trophie du lac de Buyo (sud-ouest de la Côte d'Ivoire). Etude analytique et statistique des paramètres physicochimiques et biologiques, Thèse de Doctorat $3^{\mathrm{e}}$ cycle, $210 \mathrm{pp}$. 